Submitted to ApJ, PUblished Version AT https://doi.org/10.3847/1538-4357/aacbc0

Typeset using LATEX twocolumn style in AASTeX62

\title{
Rapid Flaring in the Galactic-plane Gamma-ray Transient Fermi J0035+6131
}

\author{
DIRK PANDEL ${ }^{1}$ AND PHILIP KAARET ${ }^{2}$ \\ ${ }^{1}$ Grand Valley State University, 118 Padnos Hall of Science, 1 Campus Drive, Allendale, MI 49401, USA \\ ${ }^{2}$ The University of Iowa, Department of Physics and Astronomy, Iowa City, IA 52242, USA
}

\begin{abstract}
We investigate the gamma-ray and X-ray emission from the transient gamma-ray source Fermi J0035+6131, which was discovered with the Fermi Large Area Telescope (LAT) near the Galactic plane at $b=1.3$, and we discuss potential multi-wavelength counterparts of the gamma-ray source. Our analysis of over 9 years of Fermi LAT data revealed two flaring events lasting 10-30 hr during which the gamma-ray flux increased by a factor of $>300$ compared to the long-term average. We also analyzed X-ray data obtained with XMM-Newton and Swift and identified several sources with a hard X-ray spectrum inside the Fermi LAT confidence region. The two brightest X-ray sources have known counterparts at other wavelengths and are associated with the compact radio source VCS4 J0035+6130 and the B1 IV:nn star HD 3191, respectively. VCS4 J0035+6130, which is also detected in the near infrared, is likely an active galaxy serendipitously located behind the Galactic disk and is the most compelling candidate for the counterpart of the gamma-ray source. HD 3191 appears to be part of an $\mathrm{X}$-ray binary with a compact companion and is unlikely to be associated with Fermi J0035+6131.
\end{abstract}

Keywords: gamma rays: galaxies - X-rays: binaries - X-rays: galaxies $-\mathrm{X}$-rays: individual (VCS4 J0035+6130, NVSS J003524+613030, HD 3191)

\section{INTRODUCTION}

The Large Area Telescope (LAT) on board the Fermi Gamma-ray Space Telescope (Atwood et al. 2009) has detected numerous, previously unknown transient sources of $\mathrm{GeV}$ gamma rays. While the majority of these transient gamma-ray sources are likely extragalactic, some have been shown to be located within our Galaxy. One such Galactic gamma-ray transient is Fermi J2102+4542 (Cheung et al. 2010), which was identified as a classical nova outburst in the symbiotic star V407 Cyg (Abdo et al. 2010). This discovery established novae as a new class of gamma-ray sources, and several other novae have subsequently been detected with the Fermi LAT. Variable GeV gamma-ray emission has also been observed from several Galactic X-ray binaries such as the microquasar Cygnus X-3 (Abdo et al. 2009a) and the Highmass X-ray Binaries LS I +61 303 and LS 5039 (Abdo et al. 2009b). An investigation of Fermi LAT transients detected near the Galactic plane may reveal previously unknown $\mathrm{GeV}$ gamma-ray sources in our Galaxy.

On 2016 January 14, the Fermi LAT observed strong gamma-ray emission from the previously unknown source Fermi J0035+6131 located only 1.3 from the Galactic plane. The source was detected with a daily-averaged flux of $(5.7 \pm$ $1.5) \times 10^{-7}$ photons $\mathrm{cm}^{-2} \mathrm{~s}^{-1}$ above $100 \mathrm{MeV}$ at coordinates R.A. $=8.91$ and decl. $=61.52(\mathrm{~J} 2000.0)$ with a statistical 95\% containment radius of 0.08 (Pivato et al.
2016). The best-fit location of the gamma-ray source is 0.03 from the unidentified radio source VCS4 J0035+6130 (87GB 003232.7+611352) which was suggested as a potential counterpart. Follow-up observations of this source in the radio band 6-23 days after the gamma-ray detection indicate that, compared to earlier observations, the radio flux was higher by a factor of $1.5-2$ at frequencies above $4.8 \mathrm{GHz}$ (Trushkin et al. 2016). A near-infrared (NIR) counterpart of the radio source with magnitudes $J=16.81, H=15.64$, and $K s=14.86$ was discovered in an observation performed 14 days after the gamma-ray detection (Carrasco et al. 2016).

The region near Fermi J0035+6131 was also observed in X-rays with XMM-Newton 10 days after the Fermi LAT detection. In a preliminary analysis of the XMMNewton data, we detected two previously unknown X-ray sources inside the Fermi LAT confidence region, the radio source VCS4 J0035+6130 mentioned above and the B1 IV:nn star HD 3191 (Pandel \& Kaaret 2016; Pandel 2017). Munari \& Valisa (2016) obtained optical spectra of HD 3191 and derived its radial velocity, which was found to differ significantly from that measured several decades earlier. The hard X-ray spectrum and the apparent change in radial velocity suggest that HD 3191 may be an X-ray binary. Both X-ray sources are potential candidates for the multi-wavelength counterpart of Fermi J0035+6131.

In this paper, we present a detailed analysis of gamma-ray and X-ray data of the region near Fermi J0035+6131. We 
analyze over 9 years of Fermi LAT Pass 8 data to search for additional gamma-ray flaring activity, obtain an improved location of the transient, and constrain its spectral properties. Furthermore, we analyze X-ray data obtained with XMMNewton and Swift to identify potential counterparts of Fermi J0035+6131 and constrain their X-ray spectra and variability. We then investigate the multi-wavelength properties of these counterparts and discuss the implications for the identification of the gamma-ray transient.

\section{FERMI LAT DATA ANALYSIS}

We analyzed Fermi LAT Pass 8 data (Atwood et al. 2013) covering 9.5 years from 2008 August 4 to 2018 March 2. Events were selected in the energy range $0.1-100 \mathrm{GeV}$ from a $15^{\circ}$ radius circular region of interest centered on R.A. = 8.91 and decl. $=61.52$, the best-fit location of Fermi J0035+6131 originally reported by Pivato et al. (2016). Included in our analysis were front and back events classified as SOURCE (evclass $=128$ ) with zenith angles $<90^{\circ}$, and the Instrument Response Functions P 8R2_SOURCE_V 6 were used. The spatial, spectral, and timing analysis of the LAT data was performed using a standard unbinned maximum likelihood method with the Fermi Science Tools. ${ }^{3}$ The significance of gamma-ray sources was evaluated using the Test Statistic TS $=2 \ln \left(\mathcal{L}_{1} / \mathcal{L}_{0}\right)$ (Mattox et al. 1996), where $\mathcal{L}_{1}$ and $\mathcal{L}_{0}$ are the maximum likelihood values with and without a given source included in the model. $\sqrt{\mathrm{TS}}$ is approximately equal to the significance in terms of the standard deviation $\sigma$. In a preliminary analysis of two days of LAT data of the transient event (MJD 57401-57403) we detected four of the sources listed in the 3FGL catalog (Acero et al. 2015) inside the $15^{\circ}$ radius region of interest with a Test Statistic TS > 10: 3FGL J0007.0+7302, 3FGL J0223.6+6204, 3FGL J0240.5+6113, and 3FGL J2229.0+6114. We included these sources in our model for the likelihood analysis, but because of their comparatively low number of counts and their large distances from Fermi J0035+6131 of $>10^{\circ}$, the spectral parameters were fixed at the catalog values. Our model of the region of interest also included Galactic diffuse emission ( $\mathrm{gll} \mathrm{l}_{-\mathrm{iem}} \mathrm{V} 06$. fits) and extragalactic isotropic emission (iso_P 8R2_SOURCE_V6_V06.txt) with their normalizations as free parameters.

A gamma-ray counts map of 9.5 years of LAT data of the region around Fermi J0035+6131 is shown in Figure 1. No gamma-ray source is detected at the location of the transient, which places a $95 \%$ upper limit of $1.5 \times 10^{-9}$ photons $\mathrm{cm}^{-2} \mathrm{~s}^{-1}$ on the average gamma-ray flux between 0.1 and $100 \mathrm{GeV}$ (assuming a power-law spectrum with a photon index of -2.0 ). To search for previously undetected gamma-ray flaring activity in Fermi J0035+6131,

\footnotetext{
${ }^{3}$ https://fermi.gsfc.nasa.gov/ssc/data/analysis/
}

we divided 9.5 years of LAT data between MJD 54683 and MJD 58180 into 2-day time intervals in 1-day steps and fitted our model of the region of interest to the data of each time interval. Fermi J0035+6131 was modeled as a point source at its originally reported location having a power-law spectrum with a fixed photon index of -2.0 and variable normalization. We searched the light curve for time intervals during which the gamma-ray source is detected with a TS value $>25$, which corresponds to a $5 \sigma$ detection threshold for a single 2-day interval or a $0.2 \%$ probability of at least one false detection in the entire light curve. We found two flaring events above this threshold with TS values of 35 and 82, respectively, with the lower value corresponding to a falsedetection probability of $1 \times 10^{-5}$. The first flare occurred near MJD 57067 and had not previously been reported. The second flare, which was detected near MJD 57402, is the one reported by Pivato et al. (2016). Figure 2 shows 6-hr light curves of the gamma-ray flux and the Test Statistic TS near the times of the two flares.

To better constrain the start and end times of the two flares, we selected gamma-ray events from a $1^{\circ}$ region around Fermi J0035+6131 and used a Bayesian Blocks method (Scargle et al. 2013) with the unbinned event data. We found that the first flare lasted $\sim 10 \mathrm{hr}$ from MJD 57066.5 to 57066.9 and the second flare $\sim 30 \mathrm{hr}$ from MJD 57401.0 to 57402.2 with the first flare being three times as intense as the second. (Note that the start and end times could not be constrained to better than $\sim 0.1$ days because the object was only observable during $\sim 1 / 4$ of the 3.2-hr Fermi LAT sky survey cycle.) We then selected LAT data from the entire region of interest for the above time intervals and fit our model to the data using an unbinned maximum likelihood method. Figure 1 shows TS maps for the two time intervals obtained using gttsmap. The TS maps, which represent the significance of a putative point source at a given location, clearly show the detection of Fermi J0035+6131 with a high significance. The best-fit location of the gamma-ray source, i.e. the location with the maximum TS value, was determined using gtfindsrc. Results are shown in Table 1. To obtain stronger constraints on the source location, we also performed an analysis of the combined data from the two time intervals. Our best estimate for the location of Fermi J0035+6131 is R.A. $=8.975$ and decl. $=61.548$ with a location error of 0.056 ( $68 \%$ confidence, statistical only).

The spectral parameters of the two flares were obtained using an unbinned maximum likelihood fit with a simple power-law model with the normalization and photon index as free parameters. The average gamma-ray flux and the photon index obtained from the fit are shown in Table 1. The spectra are well described by a power-law model with a photon index near -2.0 . The number of detected gamma rays was too low to allow discriminating between different spec- 

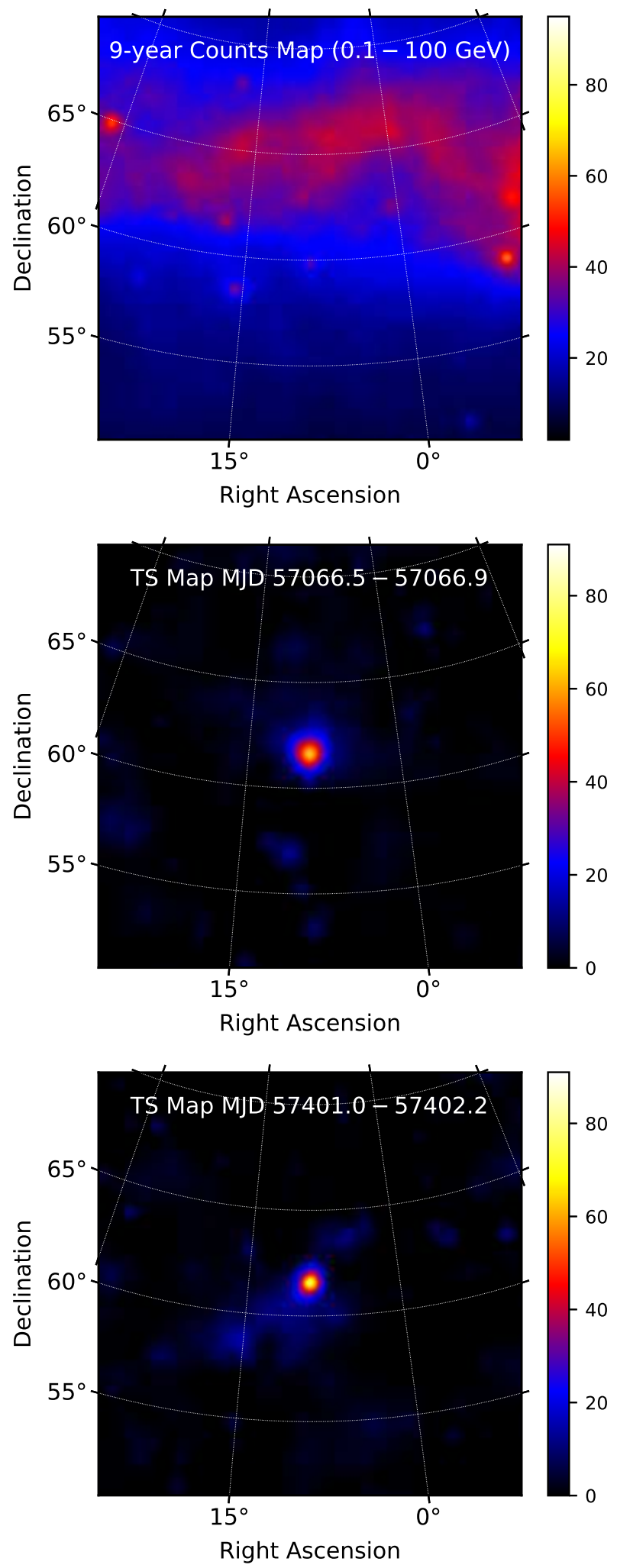

Figure 1. Smoothed 9-year gamma-ray counts map (bin size 0.05 ) of a $20^{\circ} \times 20^{\circ}$ region centered on Fermi J0035+6131 and TS maps at the times of the two flares. tral models such as broken power-law or log-parabolic models. Given the upper limit of $1.5 \times 10^{-9}$ photons $\mathrm{cm}^{-2} \mathrm{~s}^{-1}$ on the 9.5-year averaged gamma-ray flux, the two flares represent an increase in gamma-ray brightness by factors of at least 1000 and 300, respectively.

\section{X-RAY DATA ANALYSIS}

The region near Fermi J0035+6131 was observed with XMM-Newton (Jansen et al. 2001) on 2016 January 24, 10 days after the Fermi LAT detection. We obtained $11.2 \mathrm{ks}$ of data from each of the EPIC MOS cameras (Turner et al. 2001) and $9.8 \mathrm{ks}$ from the EPIC PN camera (Strüder et al. 2001). All three cameras were operated in full frame mode with the medium blocking filters. The X-ray data were filtered to include only good events with patterns $0-12$ for MOS and 0-4 for PN. For our analysis we selected X-ray events in the energy range $0.5-12 \mathrm{keV}$.

An X-ray counts map of the region near Fermi J0035+6131 is shown in Figure 3. The contours represent the confidence regions of the transient's location we obtained from our analysis of the Fermi LAT data. Six X-ray sources are detected with a likelihood DET_ML $>15$ inside the $3 \sigma(99.7 \%)$ Fermi LAT confidence region. Source locations, X-ray fluxes, hardness ratios, and likely associations for these sources are shown in Table 2.

Four of the detected X-ray sources exhibit a large hardness ratio suggesting an accreting object or other type of highenergy source. Their hard X-ray spectra make them potential candidates for the counterpart of the gamma-ray source. The two brightest of these sources are associated with the unidentified radio source VCS4 J0035+6130 and the star HD 3191, respectively. No counterparts at other wavelengths are known for the other two hard X-ray sources. The two remaining sources exhibit a very soft X-ray spectrum, and they are unlikely to be associated with the gamma-ray flare. Both have a known optical counterpart and are likely X-ray active stars.

For the two brightest sources, we fitted the X-ray spectra with various models using XSPEC (Arnaud 1996) and estimated the spectral parameter using a maximum likelihood method. In both cases, the spectra are equally well fit by either an absorbed power-law model or an absorbed bremsstrahlung model. However, an absorbed blackbody model does not provide a good fit and can be ruled out. The best-fit parameters for the power-law and bremsstrahlung models are shown in Table 3. For the other sources, the X-ray flux was too low to determine meaningful parameters from a spectral fit.

The region near Fermi J0035+6131 was also observed with the $S$ wift XRT (Burrows et al. 2005) four times between 42 and 70 days after the Fermi LAT detection with individual exposures ranging from 0.7 to $2.1 \mathrm{ks}$ in duration. Because of the lower sensitivity of the XRT compared to XMM- 

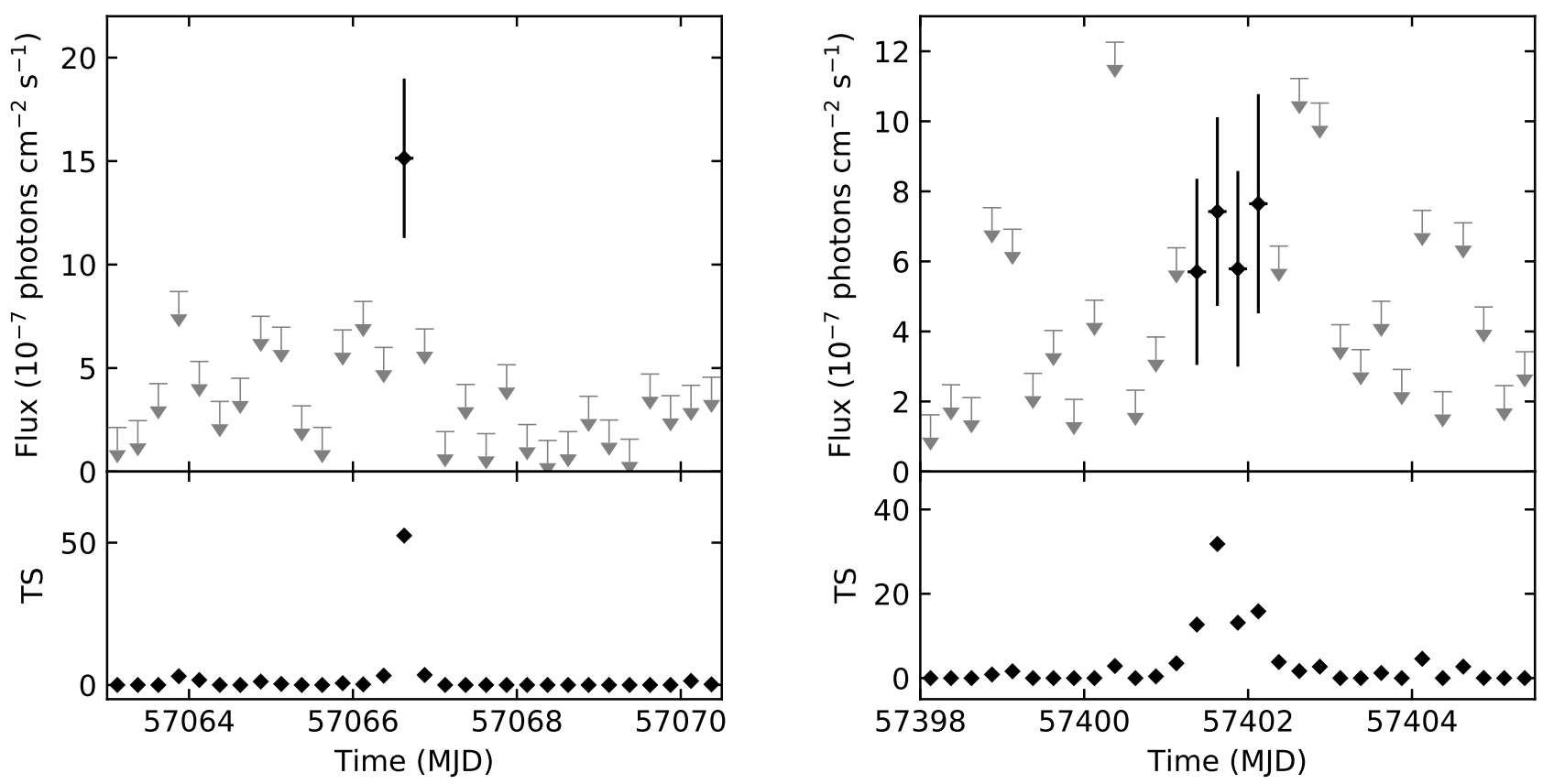

Figure 2. 6-hr gamma-ray light curves showing the flux $(0.1-100 \mathrm{GeV})$ and the Test Statistic TS of Fermi J0035+6131 near the times of the two flares. Error bars are shown at $68 \%$ confidence and upper limits at $95 \%$ confidence.

Table 1. Best-fit location, detection significance, source counts predicted by the model, and spectral parameters of Fermi J0035+6131 from our analysis of the individual and combined Fermi LAT data of the two gamma-ray flares.

\begin{tabular}{|c|c|c|c|c|c|c|c|}
\hline \multirow{2}{*}{$\begin{array}{l}\text { Time Interval } \\
\text { (MJD) }\end{array}$} & \multicolumn{2}{|c|}{ Best-fit Location } & \multirow{2}{*}{$\begin{array}{l}\text { Location } \\
\text { Error }(68 \%)\end{array}$} & \multirow{2}{*}{$\begin{array}{c}\text { TS Value } \\
\text { (Significance) }\end{array}$} & \multirow{2}{*}{$\begin{array}{c}\text { Predicted } \\
\text { Counts }\end{array}$} & \multirow{2}{*}{$\begin{array}{l}\text { Flux }(0.1-100 \mathrm{GeV}) \\
\left(\text { photons } \mathrm{cm}^{-2} \mathrm{~s}^{-1}\right)\end{array}$} & \multirow{2}{*}{$\begin{array}{c}\text { Power Law } \\
\text { Photon Index }\end{array}$} \\
\hline & R.A. & Decl. & & & & & \\
\hline $57066.5-57066.9$ & 8.993 & 61.602 & 0.110 & $71.1(8.4 \sigma)$ & 38 & $(15.1 \pm 3.7) \times 10^{-7}$ & $-2.08 \pm 0.19$ \\
\hline $57401.0-57402.2$ & 8.969 & 61.537 & 0.063 & $91.4(9.6 \sigma)$ & 55 & $(5.5 \pm 1.4) \times 10^{-7}$ & $-1.86 \pm 0.15$ \\
\hline $\begin{array}{l}57066.5-57066.9 \text { and } \\
57401.0-57402.2\end{array}$ & 8.975 & 61.548 & 0.056 & $154(12.4 \sigma)$ & 94 & $(7.7 \pm 1.4) \times 10^{-7}$ & $-1.95 \pm 0.12$ \\
\hline
\end{tabular}

Table 2. X-ray sources detected with XMM-Newton inside the $3 \sigma(99.7 \%)$ confidence region of the gamma-ray source location of Fermi J0035+6131. The source location has, in addition to the statistical error shown, a systematic error of 0. .' 5 .

\begin{tabular}{|c|c|c|c|c|c|c|c|c|}
\hline \multirow[t]{2}{*}{ ID } & \multicolumn{3}{|c|}{ Source Location } & \multicolumn{3}{|c|}{ Flux $\left(10^{-14} \mathrm{erg} \mathrm{cm}^{-2} \mathrm{~s}^{-1}\right)$} & \multirow{2}{*}{$\begin{array}{l}\text { Hardness Ratio } \\
\frac{F_{\text {hard }}-F_{\text {soft }}}{F_{\text {total }}}\end{array}$} & \multirow[t]{2}{*}{ Likely Association (Offset) } \\
\hline & R.A. & Decl. & Stat. Error & $\begin{array}{c}F_{\text {total }} \\
(0.5-12 \mathrm{keV})\end{array}$ & $\begin{array}{c}F_{\text {soft }} \\
(0.5-2 \mathrm{keV})\end{array}$ & $\begin{array}{c}F_{\text {hard }} \\
(2-12 \mathrm{keV})\end{array}$ & & \\
\hline 1 & 8.85510 & 61.50846 & $0^{\prime \prime} 2$ & 98 & 13 & 85 & 0.74 & VCS4 J0035+6130 (0.. 7) \\
\hline 2 & 8.96187 & 61.45978 & $0 . \prime 5$ & 9.5 & 1.5 & 8.0 & 0.70 & HD $3191\left(0^{\prime \prime} 1\right)$ \\
\hline 3 & 8.84006 & 61.45949 & $0 ! \prime 9$ & 3.3 & 0.3 & 3.0 & 0.80 & $\ldots$ \\
\hline 4 & 8.86784 & 61.57279 & $1{ }^{\prime \prime} 5$ & 1.14 & 0.16 & 0.98 & 0.71 & $\ldots$ \\
\hline 5 & 9.07663 & 61.51540 & $1^{\prime \prime} 2$ & 0.70 & 0.68 & 0.02 & -0.96 & USNO-B1 1515-0021314 (0.'9) \\
\hline 6 & 8.93439 & 61.48537 & $1^{\prime \prime} 3$ & 0.63 & 0.60 & 0.03 & -0.91 & USNO-B1 1514-0021151 (1."6) \\
\hline
\end{tabular}




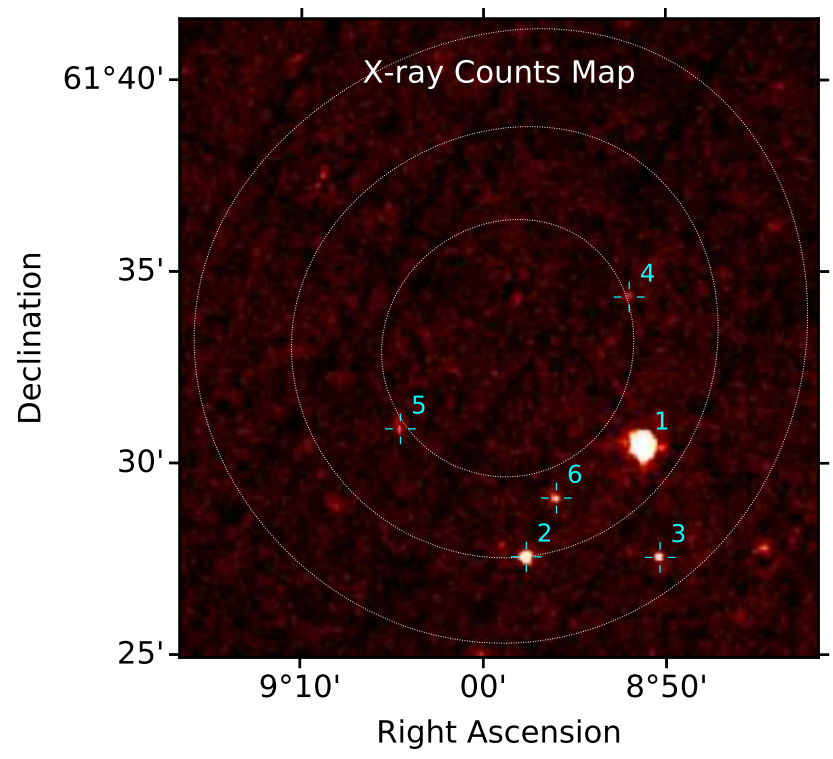

Figure 3. X-ray counts map $(0.5-12 \mathrm{keV})$ of the region near Fermi J0035+6131 obtained with XMM-Newton 10 days after the first detection of the gamma-ray transient. The contours represent the $1 \sigma, 2 \sigma$, and $3 \sigma(86 \%, 95 \%$, and $99.7 \%)$ confidence regions of the gamma-ray source location obtained from the Fermi LAT data. $\mathrm{X}$-ray sources detected inside the $3 \sigma$ confidence region are labeled with the IDs shown in Table 2).

Table 3. X-ray spectral parameters for the two brightest X-ray sources, VCS4 J0035+6130 and HD 3191, obtained by fitting two different spectral models, an absorbed power-law model and an absorbed single-temperature bremsstrahlung model, to the XMMNewton spectra. The flux shown is the absorbed flux in the 0.5-12 keV energy range, $N_{\mathrm{H}}$ is the neutral hydrogen column density (using abundances by Wilms et al. 2000), and uncertainties are shown at $90 \%$ confidence. Parameters were estimated using a maximum likelihood method with $C$ statistic (Cash 1979), and the $C$ value of the fits as well as the number of degrees of freedom (dof) are shown.

\begin{tabular}{lcc}
\hline \hline & VCS4 J0035+6130 & HD 3191 \\
\hline \multicolumn{3}{c}{ Power-law Model } \\
Flux $\left(10^{-14} \mathrm{erg} \mathrm{cm}^{-2} \mathrm{~s}^{-1}\right)$ & $103 \pm 7$ & $10.6 \pm 2.5$ \\
Photon index & $-1.57 \pm 0.10$ & $-1.5 \pm 0.4$ \\
$N_{\mathrm{H}}\left(10^{21} \mathrm{~cm}^{-2}\right)$ & $7.5 \pm 1.0$ & $4.5 \pm 3.0$ \\
$C$ value (dof) & $134(120)$ & $14.1(24)$ \\
\hline \multicolumn{3}{c}{ Bremsstrahlung Model } \\
Flux $\left(10^{-14} \mathrm{erg} \mathrm{cm}^{-2} \mathrm{~s}^{-1}\right)$ & $98 \pm 7$ & $10.1 \pm 2.7$ \\
Temperature $\left(\mathrm{keV}^{2}\right.$ & $15 \pm 5$ & $>6$ \\
$N_{\mathrm{H}}\left(10^{21} \mathrm{~cm}^{-2}\right)$ & $6.6 \pm 0.8$ & $4.1 \pm 2.2$ \\
$C$ value $(\mathrm{dof})$ & $130(120)$ & $13.9(24)$ \\
\hline
\end{tabular}

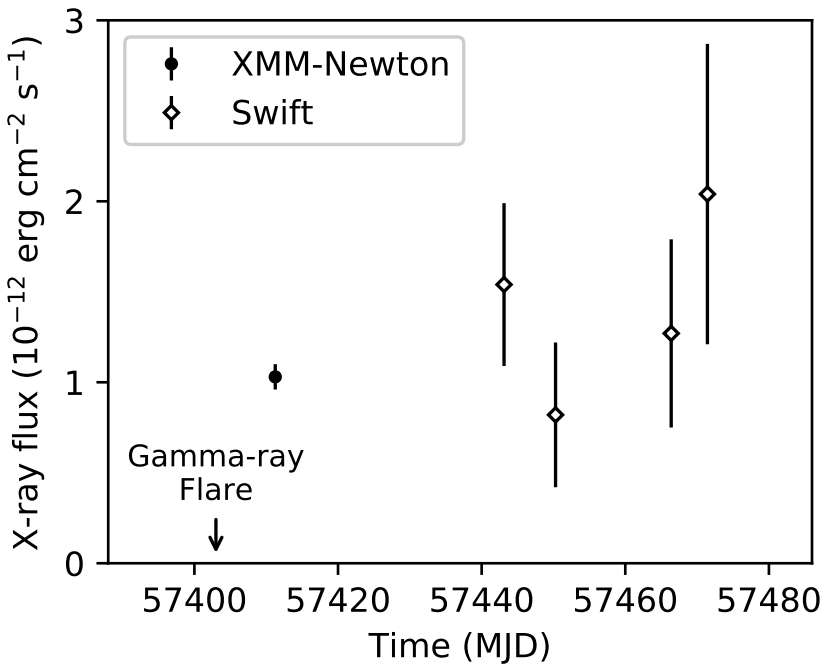

Figure 4. X-ray light curve of VCS4 J0035+6130 showing the flux in the $0.5-12 \mathrm{keV}$ energy range during the XMM-Newton and Swift observations.

Newton, we only detect the brightest X-ray source, VCS4 J0035+6130. We estimated the X-ray flux from this source for each Swift observation by fitting the spectrum with an absorbed power-law model with the photon index and neutral hydrogen column density fixed at the best-fit values obtained from the XMM-Newton spectrum (Table 3). A more detailed spectral analysis was not possible because of the low number of photons detected with the Swift XRT. A light curve of our flux measurements for VCS4 J0035+6130 is shown in Figure 4. The X-ray brightness does not appear to be strongly variable or declining as might be expected after a gamma-ray flare. However, because of their large uncertainty, the $S$ wift XRT measurements only provide poor constraints on any potential X-ray variability. For HD 3191, which was not detected with $S$ wift, we determined an upper limit of $26 \times 10^{-14} \mathrm{erg} \mathrm{cm}^{-2} \mathrm{~s}^{-1}$ on the $0.5-12 \mathrm{keV}$ flux (95\% c.1.), which is well above the flux determined from the XMM-Newton data. Therefore, no conclusions can be drawn about potential X-ray variability of HD 3191.

\section{DISCUSSION}

The high-energy processes occurring in gamma-ray emitting objects often lead to X-ray emission with a hard spectral slope. The four X-ray sources with a large hardness ratio we detect inside the Fermi LAT confidence region (Table 2) could therefore be considered potential counterparts of Fermi J0035+6131. However, for two of these sources, no counterparts are known at other wavelengths, making it impossible to classify them without further multi-wavelength observations. We therefore limit our discussion to the two brightest $\mathrm{X}$-ray sources, which have previously been detected at other wavelengths. 


\subsection{VCS4 J0035+6130}

The brightest X-ray source we detect inside the Fermi LAT confidence region is positionally coincident with the radio source VCS4 J0035+6130 (Petrov et al. 2006) whose offset of 0.7 is comparable to the error of the X-ray source location ( 0 ". 2 statistical, 0 . 5 systematic). VCS4 J0035+6130 (NVSS J003524+613030) has been observed in various radio surveys. Condon et al. (1998) measured a radio flux density of $292 \mathrm{mJy}$ at $1.4 \mathrm{GHz}$, Furst et al. (1990) a flux density of $230 \mathrm{mJy}$ at $2.7 \mathrm{GHz}$, and Gregory \& Condon (1991) a flux density of $180 \mathrm{mJy}$ at $4.85 \mathrm{GHz}$. Trushkin et al. (2016) performed radio observations of the object 6-23 days after the gamma-ray flare and measured mean flux densities of 230, 210 , and $350 \mathrm{mJy}$, respectively, at 4.8, 11.2, and $21.7 \mathrm{GHz}$. These flux densities are higher than during past observations by a factor of 1.5-2 at frequencies above $4.8 \mathrm{GHz}$, which indicates significant variability. The combined flux measurements suggest a spectral index of about -0.5 .

Pushkarev \& Kovalev (2012) analyzed VLBI data of VCS4 J0035+6130 and found that $80 \%$ of the observed radio flux is concentrated in a compact core with a size $<2$ mas (FWHM). A total flux density of $110 \mathrm{mJy}$ was measured in the VLBI maps at both 2.3 and $8.6 \mathrm{GHz}$, with $90 \mathrm{mJy}$ contained in the compact component. This is somewhat lower than the flux densities obtained from the single-dish and shorter-baseline observations mentioned above, which may indicate the presence of radio emission at angular scales larger than the VLBI field of view. The radio properties of VCS4 J0035+6130 are similar to those of the active galactic nuclei investigated by Pushkarev \& Kovalev (2012), which generally exhibit milliarcsecond sizes and spectral indices above -0.5 . The strong, compact radio emission, the flat radio spectrum, and the apparent radio flux variability suggest that VCS4 J0035+6130 is an active galaxy serendipitously located behind the Galactic disk. The extragalactic nature of the object is supported by our estimates of the neutral hydrogen column density of $N_{\mathrm{H}}=(7.5 \pm 1.0) \times 10^{21} \mathrm{~cm}^{-2}$ and $N_{\mathrm{H}}=(6.6 \pm 0.8) \times 10^{21} \mathrm{~cm}^{-2}$ (depending on the spectral model), which are somewhat larger than the total Galactic $N_{\mathrm{H}}$ along the line of sight of $5.4 \times 10^{21} \mathrm{~cm}^{-2}$ (Kalberla et al. 2005). While the larger $N_{\mathrm{H}}$ may indicate intrinsic absorption in the source, the accuracy of our $N_{\mathrm{H}}$ measurements is insufficient to draw definitive conclusions.

In follow-up observations performed 14 days after the gamma-ray flare, Carrasco et al. (2016) discovered a nearinfrared counterpart of VCS4 J0035+6130 with magnitudes $J=16.81, H=15.64$, and $K s=14.86$. The object had previously not been detected in the 2MASS survey (Skrutskie et al. 2006), but Munari \& Valisa (2016) point out that this does not necessarily imply an increase of the NIR flux. No counterpart at visible wavelength was detected in the second Palomar Observatory Sky Survey (Reid et al. 1991), which has limiting magnitudes of $B=22.5, R=20.8$, and $I=19.5$. This non-detection may not be surprising given the strong extinction of visible light from extragalactic objects near the Galactic plane. Based on the dust maps by Schlegel et al. (1998), we estimate that the total Galactic extinction in the $V$ band along the line of sight is $A_{V}=3.9 \mathrm{mag}$. This implies limits on the intrinsic brightness of the object of $B>17.4, R>17.6$, and $I>17.2$. The NIR emission experiences significantly less extinction, and the measurements by Carrasco et al. (2016) correspond to intrinsic magnitudes of $J=15.7, H=15.0$, and $K s=14.4$. Although the limits on the visual magnitudes are somewhat higher than the measured NIR magnitudes, it cannot be concluded that the object experienced an increase in brightness after the gamma-ray flare. For example, observations by Ikejiri et al. (2011) show larger visual than NIR magnitudes with differences of $V-J=1.3-2.8$ for blazars not significantly affected by extinction. Therefore, the non-detection of VCS4 J0035+6130 at visible wavelengths can be attributed to the strong extinction near the Galactic plane without presuming variability of the source.

In summary, the strong, compact radio emission with a high spectral index, the large neutral hydrogen column density, and the non-detection at visible wavelengths strongly suggest that VCS4 J0035+6130 is an active galaxy serendipitously located behind the Galactic disk. Other transient Fermi LAT sources at low Galactic latitudes have been identified as extragalactic, such as Fermi J0109+6134 (Vandenbroucke et al. 2010). Ackermann et al. (2013) found that most of the flaring gamma-ray sources detected with Fermi near the Galactic plane are probably blazars. VCS4 $\mathrm{J} 0035+6130$ is therefore the most likely counterpart of Fermi J0035+6131. However, further optical observations, in particular redshift measurements, are needed to confirm its extragalactic nature.

\section{2. $H D 3191$}

The second brightest X-ray source we detect inside the Fermi LAT confidence region is positionally coincident with HD 3191, which is classified as a B1 IV:nn star, i.e. a subgiant with very broad absorption features. The star has a visual magnitude of $V=8.6$ and an estimated distance of $\sim 2 \mathrm{kpc}$ based on a parallax measurement of $0.47 \pm 0.25$ mas (Brown et al. 2016). Our analysis of the XMM-Newton data showed that HD 3191 has an unusually hard X-ray spectrum. While early B-type stars are known to emit X-rays resulting from shocks in theirs stellar winds, they generally exhibit much softer X-rays spectra (e.g. Oskinova et al. 2011). From our spectral fit with a bremsstrahlung model, we derived a lower limit of $6 \mathrm{keV}(70 \mathrm{MK})$ on the plasma temperature, which is significantly higher than in typical early B-type stars. However, the hard spectrum can be readily ex- 
plained if HD 3191 is in fact an X-ray binary with a compact companion. This interpretation is supported by recent radial velocity measurements. Munari \& Valisa (2016) determined a heliocentric radial velocity of $-46.0 \pm 0.5 \mathrm{~km} \mathrm{~s}^{-1}$ on 2016 March 7, which differs from historical measurements by Petrie \& Pearce (1961) who found $-22 \pm 3 \mathrm{~km} \mathrm{~s}^{-1}$. Furthermore, in the optical spectrum only one stellar component is visible, which hints at a compact companion if HD 3191 is indeed part of a binary.

Based on our spectral fits, we estimate that HD 3191 has an unabsorbed, bolometric X-ray flux of $1.5 \times 10^{-13}$ erg $\mathrm{cm}^{-2} \mathrm{~s}^{-1}$ which corresponds to a luminosity of $7 \times$ $10^{31} \mathrm{erg} \mathrm{s}^{-1}$ for a distance of $2 \mathrm{kpc}$. This is significantly lower than the $10^{34}-10^{38} \mathrm{erg} \mathrm{s}^{-1}$ typically observed for High-mass X-ray Binaries (HMXBs) in which the stellar wind from an O-type or B-type star is captured by a neutron star or black hole (e.g. Shtykovskiy \& Gilfanov 2005). However, the low end of the HMXB luminosity function is not well explored, and it is conceivable that the low luminosity is due to a large orbital separation resulting in a low rate of accretion. Further observations, in particular radial velocity measurements, are needed to establish whether HD 3191 is indeed a binary with a compact companion.

Fermi has detected variable $\mathrm{GeV}$ emission from several X-ray binaries, such as the microquasar Cygnus X-3 (Abdo et al. 2009a) and the High-mass X-ray Binaries LS I +61 303 and LS 5039 (Abdo et al. 2009b). The peak gamma-ray flux observed for these binaries is comparable to the one we found for Fermi J0035+6131 (e.g. Dubus 2013, Table 2). However, their X-ray emission is generally 1-2 orders of magnitude stronger than that detected from HD 3191. E.g., Abdo et al. (2011) found flaring in the long-period gamma-ray binary PSR B1259-63 with a peak gamma-ray flux of $\sim 2 \times 10^{-6}$ photons $\mathrm{cm}^{-2} \mathrm{~s}^{-1}$ ( $\left.>100 \mathrm{MeV}\right)$, while the average X-ray flux was $\sim 10^{-11} \mathrm{erg} \mathrm{cm}^{-2} \mathrm{~s}^{-1}$, two or- ders of magnitude higher than the $1 \times 10^{-13} \mathrm{erg} \mathrm{cm}^{-2} \mathrm{~s}^{-1}$ we found for HD 3191. Furthermore, the gamma-ray emission from these binaries exhibits flaring on longer time scales than Fermi J0035+6131 (tens of days) or shows a strong orbital modulation. The gamma-ray binaries are also detected in the radio band, whereas HD 3191 does not have a known radio counterpart. It therefore seems unlikely that HD 3191 is the source of the observed gamma rays even if it can be confirmed to be an X-ray binary.

\section{CONCLUSIONS}

Considering the abundance of flaring, extragalactic gammaray sources detected with Fermi and considering the multiwavelength properties of the object, VCS4 J0035+6130 is the most likely counterpart of Fermi J0035+6131 and probably an active galaxy serendipitously located behind the Galactic disk. While the potential X-ray binary HD 3191 cannot be completely ruled out as an alternative candidate, its properties compared to known gamma-ray binaries make it unlikely to be the source of the observed gamma rays. Further observations, in particular redshift measurements for VCS4 J0035+6130 and radial velocity measurements for HD 3191, are needed to conclusively establish the nature of these objects.

D.P. acknowledges support from NASA Guest Investigator Grant 15AJ88G. This work is based on observations obtained with XMM-Newton, an ESA science mission with instruments and contributions directly funded by ESA member states and NASA.

\section{Facilities: Fermi (LAT), XMM, Swift (XRT)}

Software: Fermi Science Tools, XMM-Newton SAS, XSPEC

\section{REFERENCES}

Abdo, A. A., Ackermann, M., Ajello, M., et al. 2009a, Science,

326, 1512, doi: 10.1126/science. 1182174

—. 2009b, ApJS, 183, 46, doi: 10.1088/0067-0049/183/1/46

—. 2010, Science, 329, 817, doi: 10.1126/science.1192537

—. 2011, ApJL, 736, L11, doi: 10.1088/2041-8205/736/1/L11

Acero, F., Ackermann, M., Ajello, M., et al. 2015, ApJS, 218, 23, doi: 10.1088/0067-0049/218/2/23

Ackermann, M., Ajello, M., Albert, A., et al. 2013, ApJ, 771, 57, doi: 10.1088/0004-637X/771/1/57

Arnaud, K. A. 1996, in ASP Conf. Ser., Vol. 101, Astronomical Data Analysis Software and Systems V, ed. G. H. Jacoby \& J. Barnes (San Francisco, CA: ASP), 17
Atwood, W., Albert, A., Baldini, L., et al. 2013, ArXiv e-prints. https://arxiv.org/abs/1303.3514

Atwood, W. B., Abdo, A. A., Ackermann, M., et al. 2009, ApJ, 697, 1071, doi: 10.1088/0004-637X/697/2/1071

Brown, A. G. A., Vallenari, A., Prusti, T., et al. 2016, A\&A, 595, A2, doi: 10.1051/0004-6361/201629512

Burrows, D. N., Hill, J. E., Nousek, J. A., et al. 2005, SSRv, 120, 165, doi: 10.1007/s11214-005-5097-2

Carrasco, L., Recillas, E., Porras, A., Chavushyan, V., \& Carraminana, A. 2016, ATel, 8706

Cash, W. 1979, ApJ, 228, 939, doi: 10.1086/156922

Cheung, C. C., Donato, D., Wallace, E., et al. 2010, ATel, 2487 
Condon, J. J., Cotton, W. D., Greisen, E. W., et al. 1998, AJ, 115, 1693, doi: 10.1086/300337

Dubus, G. 2013, A\&A Rv, 21, 64, doi: 10.1007/s00159-013-0064-5

Furst, E., Reich, W., Reich, P., \& Reif, K. 1990, A\&AS, 85, 805

Gregory, P. C., \& Condon, J. J. 1991, ApJS, 75, 1011, doi: $10.1086 / 191559$

Ikejiri, Y., Uemura, M., Sasada, M., et al. 2011, PASJ, 63, 639, doi: 10.1093/pasj/63.3.327

Jansen, F., Lumb, D., Altieri, B., et al. 2001, A\&A, 365, L1, doi: 10.1051/0004-6361:20000036

Kalberla, P. M. W., Burton, W. B., Hartmann, D., et al. 2005, A\&A, 440, 775, doi: 10.1051/0004-6361:20041864

Mattox, J. R., Bertsch, D. L., Chiang, J., et al. 1996, ApJ, 461, 396, doi: $10.1086 / 177068$

Munari, U., \& Valisa, P. 2016, ATel, 8789

Oskinova, L. M., Todt, H., Ignace, R., et al. 2011, MNRAS, 416, 1456, doi: 10.1111/j.1365-2966.2011.19143.x

Pandel, D. 2017, in AIP Conf. Ser., Vol. 1792, 6th International Symposium on High Energy Gamma-Ray Astronomy, ed. F. A. Aharonian, W. Hofmann, \& F. M. Rieger (Melville, NY: AIP), 040037

Pandel, D., \& Kaaret, P. 2016, ATel, 8783

Petrie, R. M., \& Pearce, J. A. 1961, Publications of the Dominion Astrophysical Observatory Victoria, 12, 1
Petrov, L., Kovalev, Y. Y., Fomalont, E. B., \& Gordon, D. 2006, AJ, 131, 1872, doi: 10.1086/499947

Pivato, G., Buson, S., \& Razzan, M. 2016, ATel, 8554

Pushkarev, A. B., \& Kovalev, Y. Y. 2012, A\&A, 544, A34, doi: 10.1051/0004-6361/201219352

Reid, I. N., Brewer, C., Brucato, R. J., et al. 1991, PASP, 103, 661, doi: $10.1086 / 132866$

Scargle, J. D., Norris, J. P., Jackson, B., \& Chiang, J. 2013, ApJ, 764, 167, doi: 10.1088/0004-637X/764/2/167

Schlegel, D. J., Finkbeiner, D. P., \& Davis, M. 1998, ApJ, 500, 525, doi: 10.1086/305772

Shtykovskiy, P., \& Gilfanov, M. 2005, MNRAS, 362, 879, doi: 10.1111/j.1365-2966.2005.09320.x

Skrutskie, M. F., Cutri, R. M., Stiening, R., et al. 2006, AJ, 131, 1163, doi: 10.1086/498708

Strüder, L., Briel, U., Dennerl, K., et al. 2001, A\&A, 365, L18, doi: 10.1051/0004-6361:20000066

Trushkin, S. A., Nizhelskij, N. A., \& Erkenov, A. 2016, ATel, 8718

Turner, M. J. L., Abbey, A., Arnaud, M., et al. 2001, A\&A, 365, L27, doi: 10.1051/0004-6361:20000087

Vandenbroucke, J., Buehler, R., Ajello, M., et al. 2010, ApJL, 718, L166, doi: 10.1088/2041-8205/718/2/L166

Wilms, J., Allen, A., \& McCray, R. 2000, ApJ, 542, 914, doi: $10.1086 / 317016$ 